\title{
Pseudo Contact Point Monitoring for Contact State Estimation
}

\author{
Kosei KITAGAKI*, Motoyoshi FUJIWARA**, Takashi SUEHIRO*, and Tsukasa OGASAWARA*** \\ *Electrotechnical Laboratory \\ 1-1-4 Umezono, Tsukuba, Ibaraki 305-5868, Japan \\ **Mie Prefectural Industrial Research Institute \\ 5-5-45 Takachaya, Tsu-city, Mie 514-0819, Japan \\ ***Nara Institute of Science and Technology \\ 8916-5 Takayama-cho, Ikoma-city, Nara 630-0101, Japan \\ mailto: kitagaki@etl.go.jp \\ Key Words: Manipulation Skill, Contact State, Pseudo Contact Point, Force Data Processing
}

\begin{abstract}
Contact state information is indispensable for performing skillful tasks in which an end-effector should be in contact with the environment. In previous work, the authors introduced the concept of a pseudo contact point that is useful for tasks involving contact as one expression of the contact state. In this paper, an extended definition of the pseudo contact point is proposed. The pseudo contact point is defined as the foot of the perpendicular from the nearest point on a reference object to the line on which the contact point and external force vector lie. The system may estimate the contact state and its transitions by monitoring the pseudo contact point in spite of the fact that the pseudo contact point does not always denote the real contact point. Experimental results suggest the effectiveness of our method, which is particularly valuable in providing an intuitive positional description of a contact state.
\end{abstract}

\section{Introduction}

To perform skillful tasks including contact processes, a robot manipulator should be able to select suitable control laws according to the contact condition detected from force information. The simplest form of force control, popular in robot applications, keeps a particular force within a limited range, for instance by stopping a manipulators if that force exceeds $10 \mathrm{~N}$. Force data (i.e.: 3 forces and 3 moments) may include useful information related to the contact condition this is seen from the fact that a human being can make various decisions only from force data. One of the most beneficial forms of information is the position of contact a point that is useful for the accomplishment of tasks. Measuring the force data applied at this contact point, we can obtain only a line on which the contact point and the force vector lie. Therefore, other constraint conditions become necessary in order to define the point uniquely.

Some techniques which detect a point of contact by using a geometrical model of the end-effector as one of the conditions have been proposed[1]. Izumi et al. have presented a method of grinding point detection to realize a grinding robot that can recognize a grinding face and the burr shape[2]. Further, Tsujimura et al. have introduced a tactile sensing method employing force information and applied it to body shape recognition[3]. These methods are effective when the shape of the end-effector on the arm is well known.

On the other hand, in a task such as pick-and-place in which a manipulator grasps an object and releases it, the configuration of the grasped object seen from the arm is not always obtained precisely even if the shape of the object is well known. Methods to detect the contact point between the object and the environment by using more than one time of instrumentation data have been proposed for this kind of task[4],[5].

These techniques are to detect an exact contact point. However, is a strict knowledge of the contact point always necessary when working with the contact? Depending on the circumstances, where sometimes rough detection of the contact position is enough, it may not be necessary to spend either the cost of geometrical model building of the end-effector, or the time cost of multiple measurements. And also depending on the circumstances, detection of the contact state transition may be important rather than the precise position.

Previously the authors proposed a method to estimate a pseudo contact point position using a set of force and moment data in a situation in which the precise shape and configuration of the grasped object cannot be obtained[6]. In this paper, an extended definition of the pseudo contact 
point is introduced and its characteristics are presented. Pseudo contact point monitoring is a technique which detects contact conditions or transitions by monitoring the locus of the pseudo contact points.

This paper is organized by follows. In section 2 , the information which can be derived from the force data detected by a force/torque sensor attached between an arm and its end-effector is analyzed. In section 3, the pseudo contact point is defined, and the monitoring techniques are introduced. Experimental results are shown in section 4. In section 5, characteristics of our method are discussed. Finally, we summarize in section 6.

\section{Relation between a contact point and force information}

We deal with a static state and suppose that the external force applied to the grasped object is measured by a force/ torque sensor attached between the arm and the end-effector. In order to simplify the equations, the gravity term has been compensated for.

When a force is applied to one point of the grasped object as shown in Figure 1, the force vector $f_{s}$ and the moment vector $m_{s}$ detected by the force/torque sensor are as follows:

$$
\begin{gathered}
f_{s}=f_{e} \\
m_{s}=r_{e} \times f_{e}
\end{gathered}
$$

where, $r_{e}, f_{e}$ denote the contact point position vector and the external force vector. Combining equations (1) and (2), we derive

$$
m_{s}=r_{e} \times f_{s}
$$

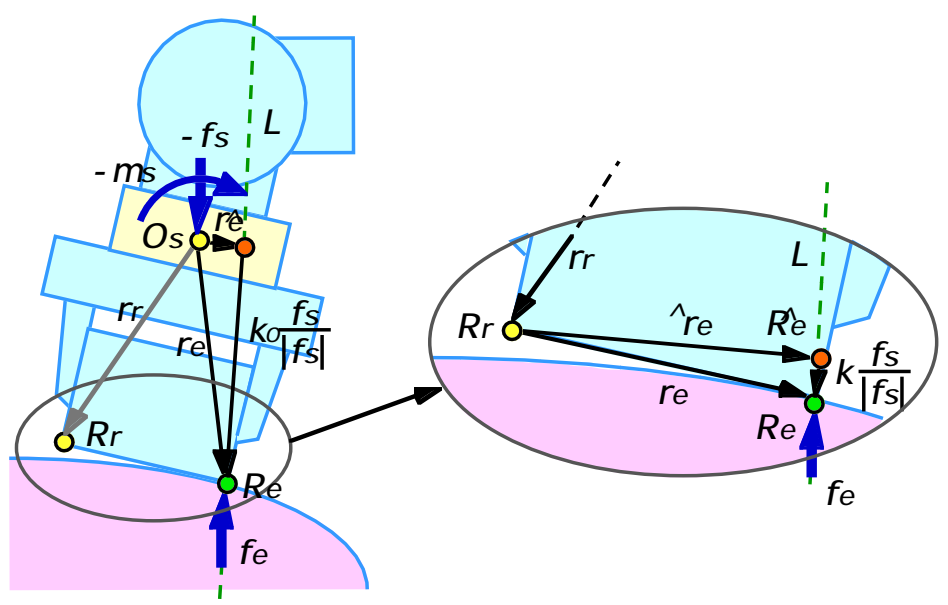

Figure 1 Relation between the force/moment detected by a F/T sensor and the external force applied on a point on an end-effector. The expanded figure shows a pseudo contact point $\left(\hat{R}_{e}\right)$ corresponding to a reference point $\left(R_{r}\right)$.
From equation (3), $r_{\mathrm{e}}$ can be written as:

$$
\begin{aligned}
& e=\hat{r_{e}}+k_{0} \frac{f_{s}}{\left|f_{s}\right|} \\
& \hat{r_{e}}=\frac{f_{s} \times m_{s}}{\left|f_{s}\right|^{2}}
\end{aligned}
$$

where, $\left|f_{s}\right|$ is the norm of $f_{s}$ and $k_{0}$ is a scalar. $k_{0}$ cannot be defined uniquely from a set of force data. Therefore, equation (4) denotes only a line on which the contact point lies (line L in Figure 1). The contact point cannot be defined uniquely from this equation, since another constraint is needs.

\section{Pseudo contact point monitoring}

\subsection{Definition of the pseudo contact point}

The pseudo contact point is defined as the nearest point on the line L to a given reference object. The contact point and the external force lie on the line L. The reference object may be permitted to be a point, a line, or a plane. Namely, when points $P_{A} \in A$ and $P_{L} \in L$ are defined by the reference object $A$ and the line $\mathrm{L}$, as follows,

$$
\forall x \in A, \forall y \in L ; \quad\left|P_{A}-P_{L}\right| \leq|x-y|
$$

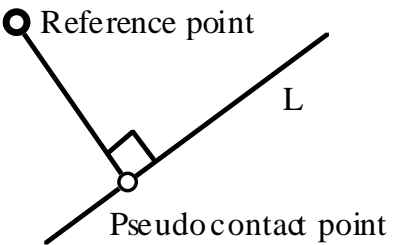

(a) Type 1. The pseudo contact point is obtained from a reference point.

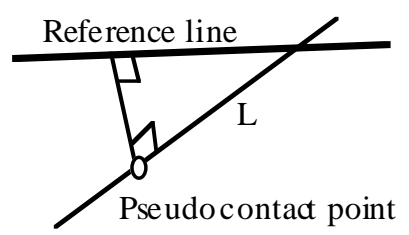

(b) Type 2. The pseudo contact point is obtained from a reference line.

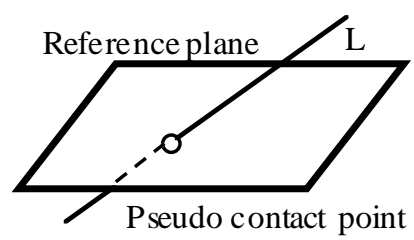

(c) Type 3. The pseudo contact point is obtained from a reference plane.

Figure 2 Typical types of pseudo contact points concerned with reference objects. 
$P_{L}$ is the pseudo contact point. The line $L$ is represented as right hand side of equation (4).

The pseudo contact points obtained with various reference objects are shown in Figure 2. Even if the line L is same, the pseudo contact point is not always in the same position; it depends on the given reference object A. A reference point, line, or plane can be specified freely according to the task objective.

Here, we derive the pseudo contact point obtained in the case in which the reference object is a point. Assuming the reference point position vector is $r_{r}$, from Figure 1 we obtain:

$$
r_{e}=r_{r}+\Delta r_{e}
$$

Rearranging by substituting from equation (3), we derive

$$
\Delta \mathrm{m}_{\mathrm{s}}=\Delta \mathrm{r}_{\mathrm{e}} \times \mathrm{f}_{\mathrm{s}}
$$

where

$$
\Delta m_{s}=m_{s}-r_{r} \times f_{s} .
$$

From equation (7) and (8), $\mathrm{r}_{\mathrm{e}}$ can be expressed as:

$$
\begin{gathered}
e=r_{r}+\Delta \hat{r}_{e}+k \frac{f_{s}}{\left|f_{s}\right|} \\
\Delta \hat{r}_{e}=\frac{f_{s} \times \Delta m_{s}}{\left|f_{s}\right|^{2}}
\end{gathered}
$$

The pseudo contact point $\hat{r}_{\mathrm{e}}$ is derived when $\mathrm{k}=0$ in equation (10), namely,

$$
\hat{r_{e}}=r_{r}+\Delta \hat{r}_{e}
$$

\subsection{Examples of the reference point setup}

A critical subject is how to set up the reference object, which must be set to be suitable for the detection objective. In this section, we present some examples of reference point establishment.

(a) Minimizing the error between the actual contact point

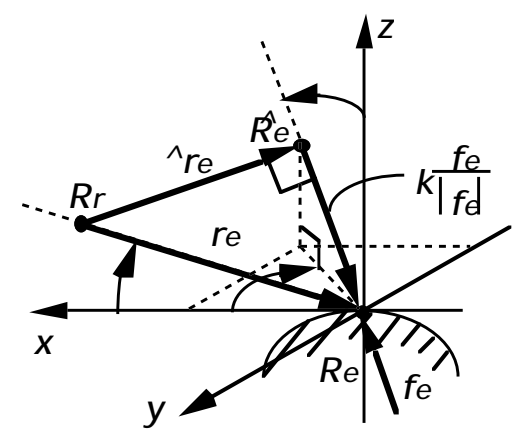

Figure 3 Geometrical analysis of the error in pseudo contact point position and the pseudo contact point

To evaluate the position error between the actual contact point and the pseudo contact point, their relationship is geometrically analyzed. We define a coordinate frame in which the $\mathrm{z}$-axis is the line normal to the surface including the contact point, and the $\mathrm{x}$ and $\mathrm{y}$ axes are defined at the reference point in the zx-plane as shown in Figure 3. In the figure, $\alpha$ is the angle between $\Delta r_{e}$ and $x$-axis, $\theta$ is the angle between $f_{S}$ and the $\mathrm{z}$-axis, and $\phi$ is the angle between the $x$-axis and a vector that is the projection of $f_{S}$ onto the $\mathrm{xy}$-plane. We obtain $\mathrm{k}$ as the following.

$$
=\left|\Delta r_{e}\right|(\cos \alpha \cos \phi \sin \theta+\sin \alpha \cos \theta
$$

Therefore, the pseudo contact point is coincident with the actual contact point when the position vector of the actual contact point seen from the reference point and the external force vector meet at right angles. On the other hand, the error grows as the angle the vectors make gets further from a right angle. The reference point is decided in consideration of this.

Let us consider the task of placing a box exactly onto the surface of table. The required information during this task is whether the bottom plane of the box is touching the table surface without any separation. Successful execution of the

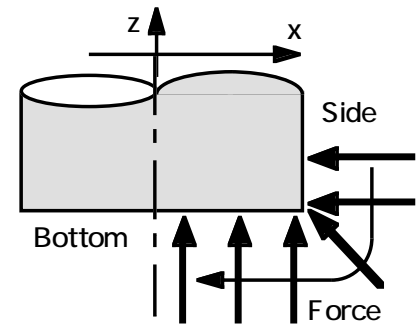

Figure 4 Continuous point contact at the side and bottom

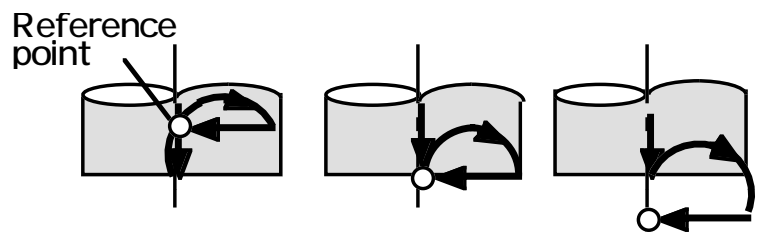

Figure 5 Trajectory of pseudo contact point
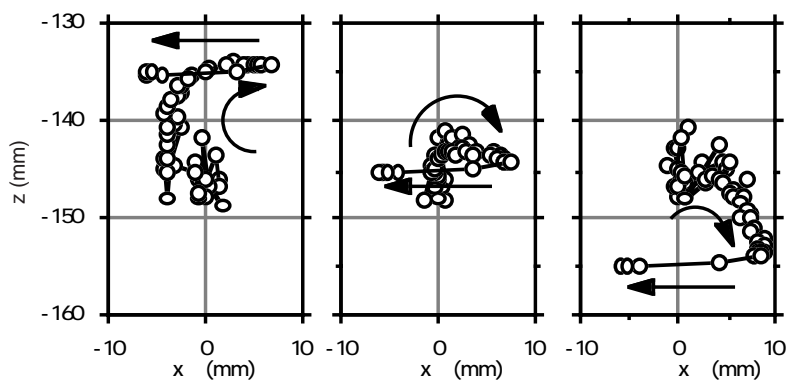

(a) Reference (b) Reference point is $(0,0$ - 0 (c) is $(0) 0$ Refence

Figure 6 Experimental trajectory of pseudo contact point 
task is indicated if the pseudo contact point is not on the contour of the bottom of the box but inside. We assume the angle that the bottom and the table surface make is small.

The pseudo contact point projected onto a plane perpendicular to the external force is coincidental with an arbitrary reference point only in the ideal case that the external force is vertically applied to the contact point. However, the variance of the pseudo contact points is big when the reference point is put far from the actual contact point in the usual case where an uncertain friction force is generated at the contact point. Consequently, the reference point should be put on the bottom of the grasped object in such cases.

\section{(b) Detecting a transition of the contact state}

In this section, reference point is set to optimize detection of a transition of contact state rather than the position of the contact point. Let us consider the task of edge detection during a motion where a cylinder is touching the environment at a point from its side to the bottom as shown in Figure 4. The theoretical trajectories of the pseudo contact points with different reference points on the axis of the cylinder are shown in Figure 5. Figure 6 shows experimental results and shows the same tendency as Figure 5. A big displacement is obtained in the $\mathrm{z}$-axis direction when a contact point moves from the side to the bottom in comparing (b) with (a) and (c). Consequently, in this work it is better to keep the reference point displaced from the bottom along the $\mathrm{z}$-axis in order to detect the transition.

\section{Experiments}

A stamping task and a bearing roller positioning task were performed to demonstrate our method using the sensor

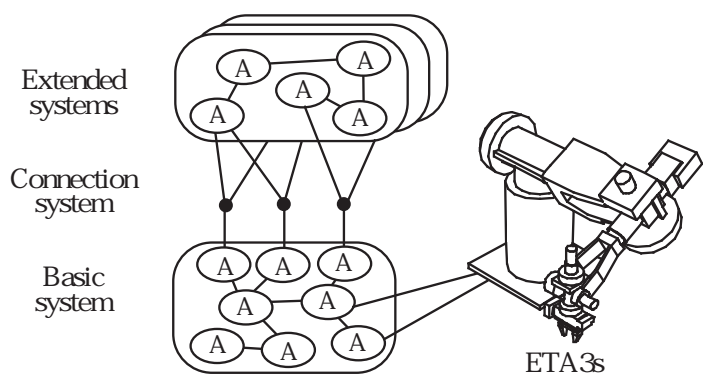

Figure 7 System configuration of TAKUMI

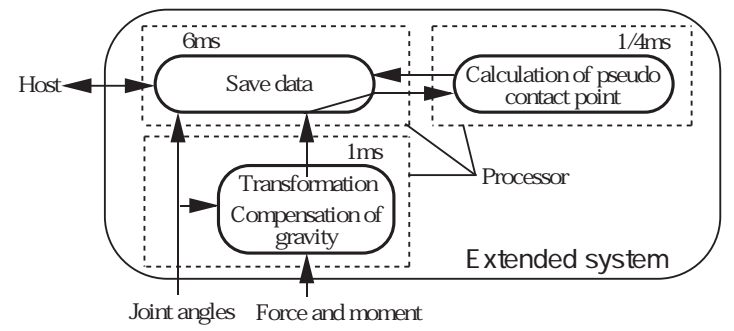

Figure 8 Pseudo contact point monitoring system based manipulation system TAKUMI[7]. TAKUMI is a system based on multi-agents using transputers, and it is composed of a direct drive manipulator ETA3s, and a control system. The control system is composed of a basic system, a connection system, and an extended system (Figure 7). The basic system has basic functions to control the manipulator such as task coordinate servoing. It can send force and joint angle data to the extended system through the connection system in $0.5 \mathrm{~ms}$, while independently servoing. The pseudo contact point monitoring system for this experiment was configured as one of the extended systems. Figure 8 shows the configuration of the monitoring system. A force/torque sensor made by Bl. Autotec, Ltd. was installed between the manipulator and its gripper. Its rated force and moment are $49 \mathrm{~N}$ and $4.9 \mathrm{Nm}$ respectively.

\subsection{Ink stamping task}

The positions of the pseudo contact points were monitored during a stamping task where the manipulator presses a grasped ink stamp onto a paper sheet on the hard rubber surface of a table. The stamp is a cylinder made of wood, with diameter $15 \mathrm{~mm}$, height $60 \mathrm{~mm}$, and weight 10 g. A scene from the experiment is shown in Figure 9. The manipulator was controlled to imitate human stamping motion. The reference point was set on the bottom of the stamp. Results are shown in Figures 10 to 12. In each figure, (a) and (b) show stamping results and path of the pseudo contact point, respectively. The pseudo contact point positions derived are the mean positions of 2 consecutive data to give continuity over time. The circular and a square continuous lines in part (b) of the figures show the reference stamp contours. Figure 10 (a) shows a successful stamping result, and (b) presents the round contour described by the locus of pseudo contact points. Figure 11 (a) is a failure

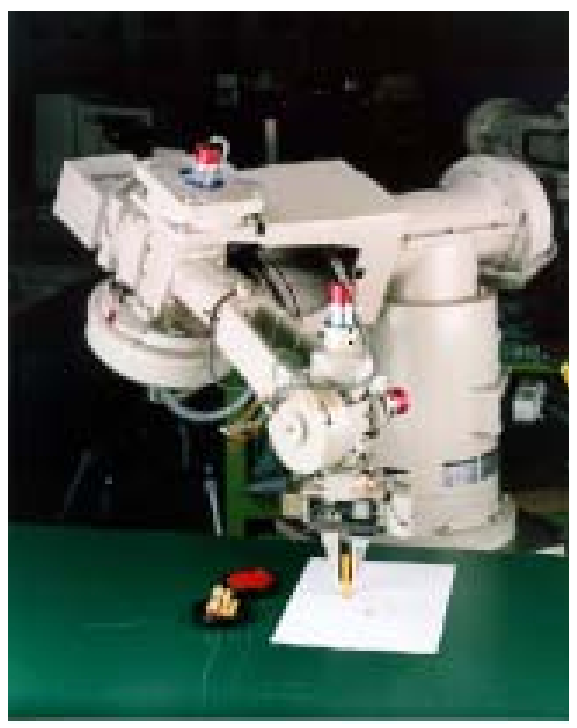

Figure 9 Experimental scene 
example when the stamp axis was slanted by 2 degrees. As for the locus shown in Figure 11 (b), it almost coincides with the stamping result. Figure 12 (a) is a successful example using a stamp which has a bottom which is square with side $15 \mathrm{~mm}$. Figure 12 (b) shows that the pseudo contact point also traced a square locus.

As for judging the success or failure of the stamping tasks, we can use the pseudo contact point locus as follows. Though the pseudo contact points were observed inside of the contour in all results, it is supposed that the contact is not an ideal point contact but a plane contact for actual stamping. On the other hand, when the pseudo contact point is never stamping inside, the center of the stamp pressed onto the paper. Stamping may interpreted as a task by pressing the whole of the contour of the stamp continuously.

\subsection{Positioning of a bearing roller for assembly[8]}

Automatic assembly of self-aligning roller bearings, a task in which a manipulator handles a roller into position for

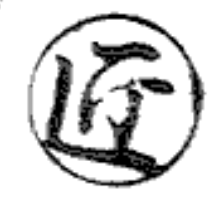

(a) Stamping result

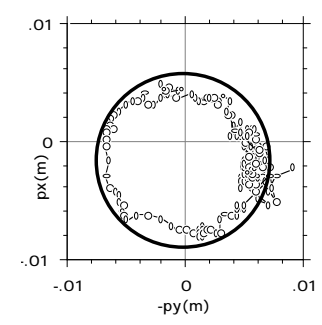

(b) Locus of pseudo contact points

Figure 10 Successful stamping result

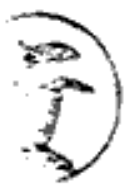

(a) Stamping result

Figure 11 Failed stamping result

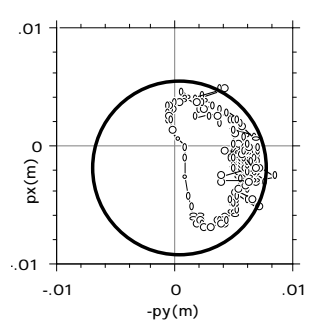

(b) Locus of pseudo contact points

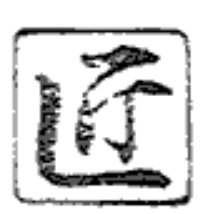

(a) Stamping result

(b) Locus of pseudo contact points

Figure 12 Successful stamping result insertion ((a) to (c) in Figure 13), was also achieved using the pseudo contact point monitoring technique. A common problem in this task is to fall into a trouble when a roller cannot be inserted because of a position error in the observation and/or its model. In this case, correction of the positioning may achieved using information derived from pseudo contact points.

A roller is to be inserted between a brim and two pillars. High positioning accuracy of the roller may not required because the roller bottom is made with radius $\mathrm{R}$, and the clearance of the inserted hole is not critical. When the center of the roller bottom is placed within the plotted area in Figure 14 (a), successful insertion without position compensation is experimentally confirmed. However, when the position of the center was out of that area, compensation is necessary for successful insertion.

We present a way to compensate the roller position using the pseudo contact point when the bottom of the roller

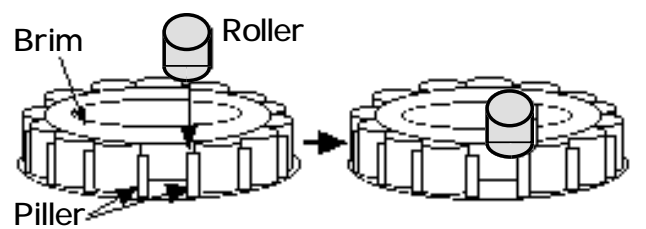

(a) A roller is moved toward (b) The roller feels the area a hole

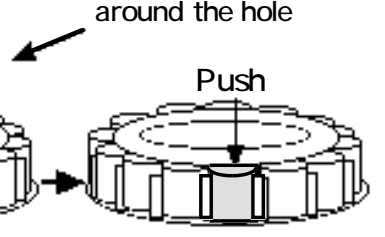

(c) A hole is probed and the roller in inserted

(d) The roller is push into the hole

Figure 13 Roller insertion task procedure

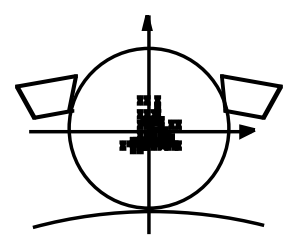

(a) Without position compensation

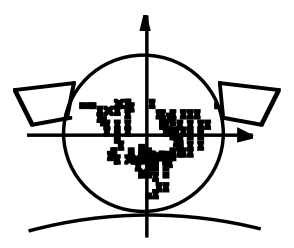

(b) With one-time position compensation
Figure 14 Positions of pseudo contact point at the first contact in successful cases of insertion

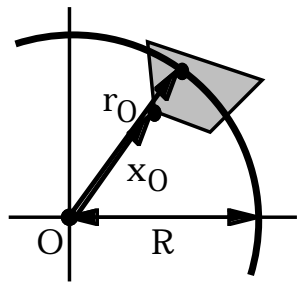

Figure 15 A simple algorithm for the compensation of roller position 
touches the brim or the pillars. Assuming that the roller radius and the pseudo contact point vector are represented by $\mathrm{R}$ and $\mathrm{x}_{0}$ as shown in Figure 15, the position vector closest to the pseudo contact point position is derived as $r_{0}=R\left(x_{0} /\left|x_{0}\right|\right)$. Therefore, to make the roller leave contact from the brim and the pillars, it should be moved by an amount $\left(x_{0}-r_{0}\right)$. Experimental results are shown in Figure 14 (b). The figure shows the result with only one-time position compensation. It shows that the area within which insertion is successful in the case using one-time compensation is wider than the area in the case without compensation. The roller diameter used in this experiment was $17 \mathrm{~mm}$.

\section{Characteristics of the pseudo contact point monitoring technique}

The benefits and drawbacks of the pseudo contact point monitoring technique are listed as follows.

(i) It allows contact condition information to be intuitively expressed as position information. For example, during a stamping task, you may easily judge whether the contour of the stamp is continuously touching the environment or not by checking simply whether the locus of the pseudo contact points draws a circle or not. Generally, it is difficult to judge this directly from the values of the observed force and moment.

(ii) A pseudo contact point is derived from one set of measured force and moment data. Therefore, the measurement time can be short, and detection of the contact state is available without obstructing objective movement.

(iii) The absolute value of the external force applied to a contact point will not affect the results because the pseudo contact point is derived using the relation of the force and the moment. Consequently, our method is robust against force noise.

(iv) Calculation is simple; the pseudo contact point is derived as the closest point on a line from the reference object.

(v) Because various real contact states are approximated to being a point contact, the contact state cannot strictly be detected. However, the contact state may be able to be detected by adding certain active sensing movements, for example varying the direction of force applied, varying the configuration of the end-effector, and so on.

(vi) There are specific dependence relations involved in each of the ways of setting up the reference object and sensing movement. Knowing the best combinations of them is necessary, and these can be thought of as sensing skills.

(vii) A precise geometric model of the end-effector and the environment should not always be necessary for qualitative detection of the contact state.

\section{Summary}

The pseudo contact point provides a new method for detecting contact states, something which is important in any robot task where a manipulator interacts with the environment. Demonstrations of a stamping task and positioning of a roller bearing have shown the possibility of various applications.

This technique means that the contact state can intuitively be represented to a human in a positional form, and this is easier to understand than raw force/moment data. Our method has wide areas application, such as the teaching of task skills, and displaying contact state information to a remote operator.

\section{Acknowledgments}

The authors express their gratitude to Dr. H. Tsukune for his encouragement and support of this work, and to the members of the Robotic Group at the Electrotechnical Laboratory for their valuable comments.

\section{References}

[1] J. K. Salisbury, "Interpretation of Contact Geometries from Force Measurements," In M.Brady and R.Paul, Proc. 1st Int. Symp. on Robotics Research, MIT Press, pp.565-577, 1984.

[2] T. Izumi, T. Narikiyo, and Y. Fukui, "Instructionless grinding robot depending on three force information," Adv. Robot., Vol.2, No.1, pp.55-67, 1987.

[3] T. Tsujimura and T. Yabuta, "Object Detection by Tactile Sensing Method Employing Force/Torque Information," IEEE Trans. Robotics and Automation, Vol.5, No.4, pp.444-450, 1989.

[4] K. Nagata, T. Ogasawara, and K. Takase, "Pose Estimation of Grasped Object from Contact Force or Joint Data of Manipulator," Trans. Soc. Instrum. Control Eng., Vol.28, No.7, pp783-789, 1992. (in Japanese)

[5] K. Kitagaki, T. Ogasawara, and T. Suehiro, "Contact State Detection by Force Sensing for Assembly Tasks," 1994 IEEE Int. Conf. on Multisensor Fusion and Integration for Intelligent Systems, pp.366-370, 1994.

[6] K. Kitagaki, T. Suehiro, and T. Ogasawara, "Monitoring of a Pseudo Contact Point for Fine Manipulation," Int. Conf. on Intelligent Robots and Systems (IROS'96), Vol.2, pp.757-762, 1996.

[7] K. Kitagaki, T. Suehiro, T. Ogasawara, and Y. H. Liu, "Sensor Based Parallel Processing Manipulation System: TAKUMI," J. of the Robotics Soc. of Japan, Vol.15, No.3, pp.363-372, 1997. (in Japanese)

[8] M. Fujiwara, K. Kitagaki, and T. Ogasawara, "Positioning for Bearing Roller Insertion using Pseudo Contact Point Position," 16th Conf. on Robotics Soc. of Japan, Vol.3, pp.1191-1192, 1998. (in Japanese) 